side-effects should be eliminated wherever possible, and the value of pyridoxine in treating depression should be studied further. Meanwhile doctors should be careful about prescribing oral contraceptives for anyone with a clear-cut history of depression.

\section{Urinary Symptoms in General Practice}

In domiciliary practice 12 per 1,000 consultations are on account of symptoms suggesting infection of the urinary tract. ${ }^{1}$ This figure underestimates the morbidity from urinary-tract disease in the population, since only $40 \%$ of patients with urinary symptoms consult their doctor ${ }^{2}$ and also because the figure refers solely to symptoms of infection.

In this issue Dr. J. Steensberg and his colleagues report the prevalence of urinary-tract disease of all kinds among 22,000 adults aged 16 and over who attended ten general practitioners in a suburban area of Copenhagen over a period of one year. They discovered 741 cases of urinary-tract disease, which amounted to 34 per 1,000 consultations in the year. Women outnumbered men by 3 to 1 . As might have been expected, the commonest complaints in both sexes were frequency, dysuria, urgency, and foul-smelling urine. In $54 \%$ of the women and $56 \%$ of the men with these complaints evidence of urinary infection was obtained, but in the remainder evidence for infection was doubtful or non-existent. This observation confirms the findingś of D. J. A. Gallagher and colleagues ${ }^{3}$ and N. C. Mond, ${ }^{4}$ who showed that only half of all women with symptoms suggesting urinary infection had a positive urine culture.

Dr. Steensberg and colleagues' data allow a comparison to be made among patients with symptoms of urinary-tract infection between those with an infected urine and those in whom the urine was not infected. Women in whom the urine was sterile were younger and their symptoms were more frequently those of "cystitis" than upper urinary-tract disease. In the non-infected women vaginal discharge and vaginitis were more frequently encountered than in the infected group, whereas genital prolapse was a common finding in the older infected women. Marital status did not affect the prevalence of symptoms in the uninfected group, whereas symptoms among the infected women were more common among judicially separated, divorced, and married women than among spinsters. Proteinuria, leucocyturia, and haematuria were all more commonly encountered in the infected group. Among the males symptoms of urinary-tract infection in the absence of significant bacteriuria were common in the younger age groups, whereas infected urines were frequently noted in old men with enlarged prostates.

These observations contribute to our understanding of the urethral syndrome-the syndrome of dysuria, frequency, and urgency in the absence of an infected urine. It is clear that

\footnotetext{
1 Fry, J., Dillane, J. B., Joiner, C. L., and Williams, J. D., Lancet, 1962, $1,1318$.

Danish National Morbidity Survey, The Sickness Survey of Denmark, 1951-1954, 1960. Copenhagen, Munksgaard.

Gallagher, D. J. A., Montgomerie, J. Z., and North, J. D. K., British Medical fournal, 1965, 1, 622 .

4 Mond, N. C., Proceedings of the Royal Society of Medicine, 1964, 57, 1119.

5 Meares, E. M., and Stamey, T. A., Investigative Urology, 1968, 5,

- British Medical fournal, 1968, 2, 192.
}

this disorder is sometimes caused by a primary disorder of the genital organs. Furthermore, this puzzling syndrome does not appear to be confined to women, since bacteriuria was not found in $44 \%$ of all the men presenting with symptoms of urinary infection. It has been claimed that in males the syndrome results from chronic bacterial prostatitis ${ }^{5}$ and in females from infection of the para-urethral glands. ${ }^{6}$ But the urethral syndrome probably has a variety of causes, since some patients can be relieved of their symptoms by avoiding such precipitating factors as cold, excessive trauma during sexual intercourse, antiseptic douches, deodorants, bubble baths, contraceptive foam, intrauterine devices, and rubber, to which they may have sensitivity reactions. But most patients find relief of symptoms difficult to achieve.

The findings of Dr. Steensberg and his colleagues also draw attention to the frequency of urolithiasis in middle-aged men and women. The rate for men was 3 per 1,000 consultations per annum as compared with 1 per 1,000 for the women. These observations underline the need for radiological investigation of patients presenting with urinary symptoms, since urolithiasis may be amenable to treatment. Diseases other than those mentioned above were much more rarely encountered during the period of the survey. Only four cases of bladder papillomata and two of bladder carcinoma were seen among the 741 persons with urinary tract disease. Dr. Steensberg and his colleagues have performed a service by once again focusing attention on the urethral syndrome as a common source of morbidity. Their observations are a stimulus to further research in this field.

\section{Treatment with Neutrons}

The neutron generator is the latest in a long series of machines devised by nuclear scientists and adapted for radiotherapy. The cyclotron at Hammersmith Hospital has been used experimentally over several years, ${ }^{1}$ but a specially built neutron therapy machine developed by co-operative effort from users, manufacturers, and Government departments will be installed at the Christie Hospital, Manchester, next summer and will probably be in full use early in 1971 .

In penetrating power the neutrons to be used for cancer therapy are comparable with $250 \mathrm{kV} x$ rays of a generation ago, but their potential efficacy depends on other factors. Anoxic cells, which may exist in the centre of a tumour, are more than ordinarily resistant to damage by radiation. This observation is the basis for the use of hyperbaric oxygen in radiotherapy, a technique still undergoing clinical trial. ${ }^{23}$ The protective effect of cellular anoxia is distinctly less with neutrons than with $x$ rays, and it is for this reason that neutron therapy is now being studied. The technical problems are formidable but appear to be soluble ; the equipment to be installed in Manchester will be comparable in size and cost

\footnotetext{
' Morgan, R. L., in Modern Trends in Radiotherapy, vol. 1, ed. T. J. Deeley and C. A. P. Wood, p. 171, London, Butterworths, 1967. Churchill-Davidson, I., et al., British fournal of Radiology, 1966, 39, 321 .

3 Brenk, H. A. S. van den, American fournal of Roentgenology, 1968, $102,8$.

Thomlinson, R. H., in Modern Trends in Radiotherapy, vol. 1, ed. T. J. Deeley and C. A. P. Wood, p. 52. London, Butterworths, 1967.

5 Field, S. B., Jones, T., and Thomlinson, R. H., British fournal of Radiology, 1968, 41, 597.

- Cheshire, P. J., and Lindop, P. J., British fournal of Radiology, 1969,
} 42,215 . 
with one of the linear accelerators already familiar in radiotherapy departments.

Some unsolved biological problems remain. Small early tumours are probably adequately nourished and unlikely to contain anoxic cells. What is not known is whether a tumour large enough to have an anoxic core will already have initiated metastatic spread and so be beyond the reach of effective treatment by local irradiation. But it is possible that anoxic cells are destroyed to some extent by existing techniques with high-energy $x$ rays. The total radiation dose is usually delivered in daily fractions over a period of three to four weeks. The treatment does more than merely incapacitate adequately oxygenated malignant cells. After each fractional dose of radiation some anoxic cells become reoxygenated and become more sensitive to subsequent attack. $^{4-6}$ In these circumstances the correct fractionation regimen required for neutron therapy remains to be established by further experiment.

Radiation is a comparatively crude instrument for tackling the complex challenge presented by cancer, but neutron therapy, used with scientific insight, may add to the basic knowledge needed for therapeutic advances.

\section{Psychogenic Dyspnoea}

The diagnosis of psychogenic dyspnoea is too often based on a failure to find organic cause for the breathlessness. A more positive approach to this problem has now been made by B. H. Burns and J. B. L. Howell, who carried out a careful psychiatric assessment of 62 patients attending a clinic for respiratory diseases. In 31 of these patients dyspnoea was regarded as disproportionate in relation to the severity of organic disease. The remaining 31 were considered to have a degree of organic disease appropriate to their complaint of breathlessness, and these served as a control group.

A comparison of the two groups showed that both a past and family history of mental illness were more frequent among the patients with "disproportionate" dyspnoea. Recent bereavement, marital dysharmony, iatrogenic influences, potential gain from illness, and absence from work were also recorded more often in this group. The patient's account of his breathlessness differed between the two groups, those with "disproportionate" dyspnoea complaining of difficulty in breathing in rather than out and of episodic dyspnoea, especially during the night or while talking. Dyspnoea was more closely related to the emotional environment than to effort and could often be relieved by sedatives or alcohol. Other features characteristic of this group included sighing and other abnormal breathing patterns, noisy respiration and hyperventilation resulting in hypocapnia, tetany, cramps, fainting attacks, tightness in the chest, angor animi, paraesthesiae, palpitations, and dryness of the mouth. These symptoms had often led to extensive hospital investigations to exclude conditions such as epilepsy, angina, and thyrotoxicosis.

Ultimately it was possible to make a definitive psychiatric diagnosis in all the patients with disproportionate dyspnoea and to classify their illness as depressive or hysterical or an anxiety reaction. Suitable psychotherapy produced symptomatic improvement in all patients except for those with

1 Burns, B. H., and Howell, J. B. L., Quarterly fournal of Medicine, 1969, 38, 277. hysterical reactions. This improvement was sustained over a two- to three-year period of observation, and during this time there had been no deterioration in lung function.

It should be noted that Burns and Howell used patients with severe airways obstruction as their controls. In such cases the organic nature of the disease is usually obvious to relatives and doctors, so that both sympathy and active therapy are readily available. Unfortunately, this does not apply to all forms of organic dyspnoea. For example, in the early stages of fibrosing alveolitis and of recurrent pulmonary embolism breathlessness may be "disproportionate" in relation to clinical, radiological, and spirometric evidence of disease. The suspicion of malingering may then arise and so damage the relationship between the patient, his family, and his medical adviser that a true psychiatric illness may ensue. Moreover, these patients can display some of the most characteristic symptoms of Burns and Howell's " psychogenic" group. Episodic dyspnoea at rest with tightness in the chest, sweating, palpitations, faintness, and angor animi may result from pulmonary embolism as well as from hyperventilation, while hyperventilation itself is a well-recognized feature of fibrosing alveolitis. Another difficulty arises from the fact that hypoxia can cause mental symptoms and still further confuse the psychiatric assessment.

Burns and Howell did not record the arterial oxygen tension of their patients (except in three cases) but confined their physiological measurements to the vital capacity, forced expiratory volume, and $\mathrm{PCO}_{2}$. These tests, together with clinical radiological and psychiatric assessment, proved sufficient to distinguish their patients with "psychogenic" dyspnoea from those with severe airways obstruction. The separation of patients with psychogenic and organic dyspnoea might well have been less distinct if those with dyspnoea due to causes other than airways obstruction had been studied. More comprehensive tests of lung function (to include arterial oxygen tension and the gas transfer factor) are then needed to detect organic disease, and these should invariably be done before dyspnoea is attributed to psychogenic causes.

\section{Immunological Aspects of Addison's Disease}

Most patients with Addison's disease seen at the present time have idiopathic atrophy of the adrenal glands. This form of the disease is known to have clinical associations with thyroiditis, as in Schmidt's syndrome, ${ }^{1}$ or with other autoimmune diseases, including pernicious anaemia, thyrotoxicosis, idiopathic hypoparathyroidism with or without moniliasis, ${ }^{2}$ and also diabetes mellitus, in which autoimmunity has not been detected. Histologically the adrenal lesions ${ }^{3}$ bear a striking resemblance to the thyroid atrophy of myxoedema and to the atrophic gastritis of pernicious anaemia. The cortical epithelial cells undergo destruction, with attempts at regeneration, and are surrounded with lymphoid cells with occasional follicles and plasma cells, while the medulla is spared. Immunization of animals with adrenal extracts and Freund's adjuvant leads to an adrenalitis resembling the human condition. ${ }^{4-6}$

Adrenal antibodies were first reported in 1957 by J. R. Anderson and colleagues ${ }^{7}$ in the serum of a patient who suffered from Hashimoto's disease and later developed adrenal 